\title{
Effect of Yttrium (Y) and Zirconium (Zr) Doping on the Thermodynamical Stability of the Cubic $\mathrm{Ba}_{0.5} \mathrm{Sr}_{0.5} \mathrm{Co}_{0.8} \mathrm{FeO}_{2} \mathrm{O}_{3-\delta}$ Phase
}

\author{
M. Meffert ${ }^{1}$, P. Müller ${ }^{1}$, H. Störmer ${ }^{1}$, L.-S. Unger ${ }^{2}$, C. Niedrig ${ }^{2}$, S. F. Wagner ${ }^{2}$, S. Saher ${ }^{3}$, H. \\ Bouwmeester $^{3}$, E. Ivers-Tiffée ${ }^{2}$, D. Gerthsen ${ }^{1}$. \\ ${ }^{1 .}$ Laboratory for Electron Microscopy (LEM), Karlsruhe Institute of Technology (KIT), 76131 \\ Karlsruhe/Germany \\ ${ }^{2}$ Institute of Materials for Electrical and Electronic Engineering (IWE), Karlsruhe Institute of \\ Technology (KIT), 76131 Karlsruhe/Germany \\ ${ }^{3 .}$ Inorganic Membranes, MESA+ Institute of Nanotechnology, Faculty of Science and Technology, \\ University of Twente, 7500 Enschede/The Netherlands
}

Mixed ionic-electronic conducting (MIEC) ceramic materials are currently considered as membranes for oxygen production due to their oxygen-ion and electronic conductivity. Among other MIECs $\mathrm{Ba}_{0.5} \mathrm{Sr} 0.5 \mathrm{Co}_{0.8} \mathrm{Fe}_{0.2} \mathrm{O} 3-\delta$ (denoted BSCF) possesses superior oxygen permeation properties due to its high oxygen non-stoichiometry. However, the inherent instability of the cubic phase during long-time operation in the temperature range of $700-800{ }^{\circ} \mathrm{C}$ is a major drawback. Below $825{ }^{\circ} \mathrm{C}$, a gradual decline in oxygen permeation over time is associated with partial decomposition of the cubic phase into a hexagonal phase $[1,2]$. The formation of the hexagonal phase can be correlated with the increase of the oxygen concentration at lower temperatures, which requires an increase of the average valence state for the multivalent Co-cations [3]. TEM studies also revealed additional secondary phases in BSCF with low crystal symmetry and plate-like morphology. These plate-like phases consist of lamellar stackings of the cubic, hexagonal and $\mathrm{Ba}_{n+1} \mathrm{ConO}_{n+3}(\mathrm{Co} 8 \mathrm{O} 8)(\mathrm{n} \geq 2)$ phase [3]. Moreover, the Co-cation in BSCF has a high tendency to diffuse out of the cubic lattice forming $\mathrm{CoO}$ precipitates which might act as nuclei for the lamellar phases. To counteract the cation valence change and, hence, decomposition of the cubic phase B-site doping with monovalent transition metals was proposed. Recent studies have shown beneficial effects for doping with $\mathrm{Y}$ or $\mathrm{Zr}$ [4,5]. However, the impact regarding the microstructure is not well understood yet. Therefore, an extensive SEM and TEM study was performed on Y- and Zr-doped BSCF which includes the variation of numerous parameters like sintering temperature, annealing temperature and dopant concentration. To verify that the dopant is incorporated on the desired B-type lattice site, the technique of atom location by channeling enhanced microanalysis was used [6].

Secondary phase formation is strongly influenced by the availability of nucleation sites. In undoped BSCF the hexagonal phase is observed at grain boundaries and plate-like precipitates which can be observed in SEM images after mechanical polishing and etching with a colloidal silica suspension (Fig. 1). The plate-like phase seems to nucleate at grain boundaries and $\mathrm{CoO}$ precipitates. However, in $\mathrm{Zr}$-and Y-doped BSCF the plate-like phase is strongly suppressed, confining the hexagonal phase to grain boundaries even at dopant concentrations as low as $3 \mathrm{at} \%$. In addition Y-doping prevents the formation of $\mathrm{CoO}$ precipitates. Grain size is an important parameter regarding the formation of secondary phases because they are nucleation controlled. Y- and Zr-doped samples with increased grain-size reveal only small amounts of the hexagonal phase at grain boundaries even after long time operation at high temperature. 
Chemical analysis of the hexagonal phase revealed an elevated Co-concentration and strong depletion of iron and dopant elements (Fig. 2 for an Y-doped BSCF sample). This can be rationalized by the available space for B-site cations in the hexagonal lattice which is insufficient for larger ions like Fe, Y, $\mathrm{Zr}$. The depletion of Fe and $\mathrm{Y}$ in the hexagonal phase and their slight enhancement in the adjacent cubic phase is observed in the EDXS maps in Fig. 2. Hence, the required out-diffusion of the dopants is assumed to slow down the decomposition and is therefore thought to be responsible for the improved stability of the cubic phase. EELS was employed to determine the valence states of the multivalent cations. For Co the white-line distance of the $\mathrm{L}_{2,3}$ peaks was evaluated by a new valence state mapping technique proposed by our group [7]. The valence of Co changes from $2.0+$ in the cubic phase to $2.6+$ in the hexagonal phase (Fig. 3).

\section{References:}

[1] S. Švarcová et al, SSI 178 (2008) p. 1787.

[2] C. Niedrig et al, SSI 197 (2011) p. 25.

[3] P. Müller et al, Chem. Mater. 25 (2013), p. 564.

[4] S. Yakovlev et al, Appl. Phys. Lett. 96 (2010) p. 254101.

[5] P. Haworth et al, Sep. Purif. Technol. 81 (2011) p. 88.

[б] J. Tafto and J. Spence, Science 218 (1982) p. 49.

[7] P. Müller et al, Microsc. Microanal. 19 (2013) p. 1595.

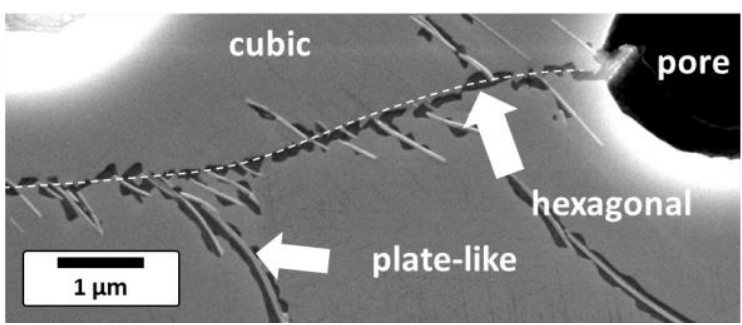

Figure 1. SEM image of undoped BSCF with plate-like phase (light grey) and hexagonal phase (dark grey). Dashed white line corresponds to grain boundary.
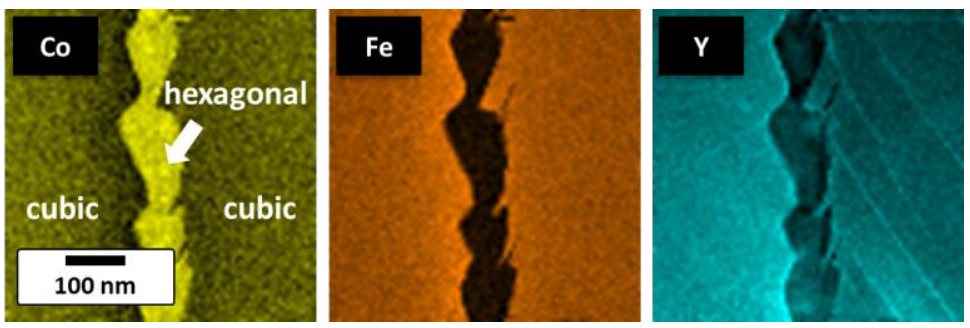

Figure 2. EDXS mapping of $\mathrm{Co}, \mathrm{Fe}$ and $\mathrm{Y}$ at a grain boundary in $3 \mathrm{at} \% \mathrm{Y}$ doped BSCF

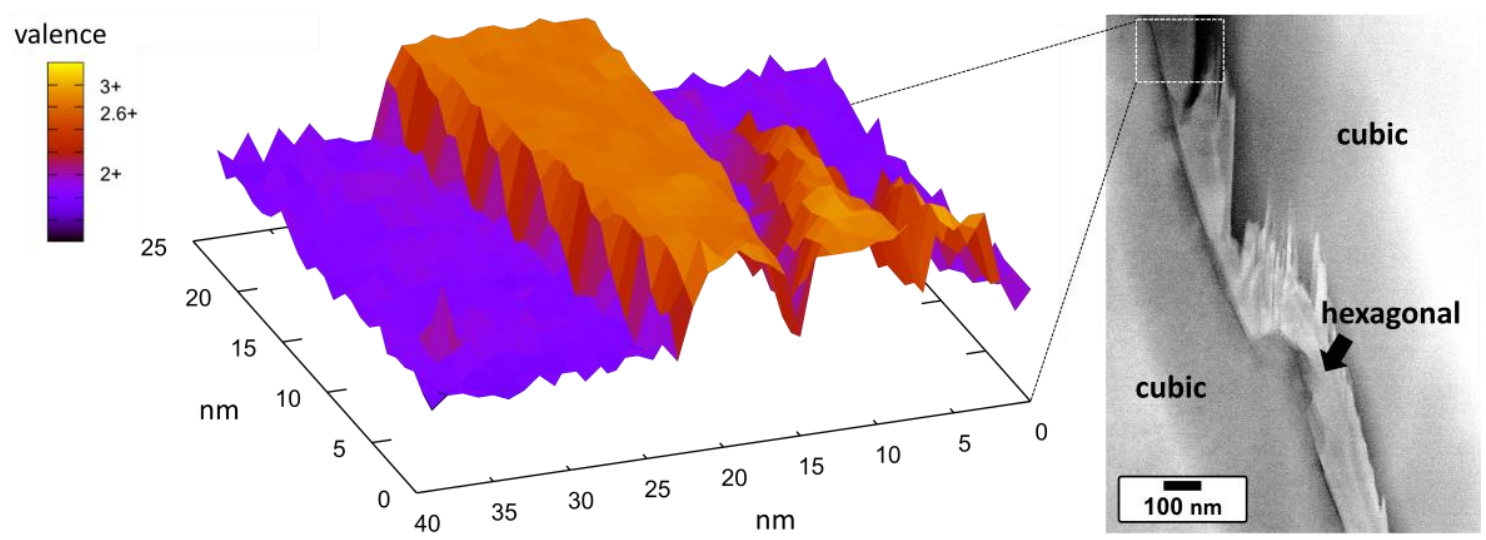

Figure 3. Valence mapping of $\mathrm{Co}$ at a grain boundary decorated with hexagonal phase in 3 at $\% \mathrm{Y}$ doped BSCF annealed for $100 \mathrm{~h}$ at $730{ }^{\circ} \mathrm{C}$ 